\title{
Enseñar a pensar: Del juego al razonamiento matemático
}

\author{
Miguel Delgado Pineda, Adoración Medina Albós \\ Departamento de Matemáticas Fundamentales, \\ Universidad Nacional de Educación a Distancia (UNED), \\ miguel@mat.uned.es, marmedina@madrid.uned.es \\ Presentado en eXIDO16 (2016)

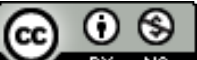

\begin{abstract}
Resumen
En este trabajo presentamos una experiencia innovadora que se desarrolla en el curso Matemáticas Recreativas en el Aula de Matemáticas del Programa Formación del Profesorado de la UNED
\end{abstract}

Los inscritos en el curso pueden comprobar el potencial didáctico que tiene utilidad actividades de Matemática Recreativa. Además, deben formular futuras propuestas innovadoras propias incorporando algunas actividades recreativas en la materia que imparten. No es fácil integrar de forma coherente las actividades de recreativas con los contenidos curriculares de una materia, por ello aprender a realizar esta tarea es el objetivo principal, por tanto aprenden a diseñar y plantear propuestas de innovación docente donde algunas innovaciones están relacionadas con las Matemática Recreativa.

El objetivo principal al que dificultad enfrenta el profesor de matemáticas es enseñar a pensar a los estudiantes y que los estudiantes aprendan a hacer eso. Somos conscientes que eso no es una tarea sencilla, pues la tarea consiste esencialmente en transitar de la simple presentación de la resolución mecánica de ejercicios, o resolución algorítmica, a enfrentar al estudiante al análisis de auténticos problemas matemáticos y que los entienda. Esos problemas en los que es preciso relacionar contenidos diversos, proceder a razonar en profundidad mediante la observación de las condiciones del problema y, además, es necesario aplicar alguna estrategia de resolución de problemas que debe ser elegida entre varias potencialmente utilizables. Entendemos que utilizar actividades inmersas en la Matemática Recreativa ofrece un marco de trabajo ideal para que el docente pueda instruir a los estudiantes en ese tránsito. Entendemos la Matemática Recreativa va más allá de su componente lúdico y motivador es un buen medio transversal a todo el conocimiento matemático, y es una auténtica herramienta didáctica para afrontar la enseñanza de las Matemáticas. Por tanto, asumimos el reto de enseñar a los docentes inscritos a utilizarla como puente para desarrollar el pensamiento matemático.

Palabras clave: Matemática Recreativa, Formación del Profesorado, Didáctica de la Matemática, Resolución de Problemas, Innovación Docente.

\section{Introducción}


Casi todos los libros de juegos matemáticos se centran en la vertiente lúdica de la mis la Matemática Recreativa, MR, sin embargo entendemos que tiene un notable potencial didáctico dentro del aula. Por ello, se debe considerar a la Matemática Recreativa como algo más que un conjunto de juegos, acertijos, enigmas o desafíos para entretener a los estudiantes. Pero debemos observar que el profesor que pretenda introducir la matemática recreativa en el aula, debe ser consciente de que esto no es una tarea ingenuamente sencilla, pues debe intentar emplearla como herramienta educativa y en ese intento se encontrará con la falta de estímulo de su entorno académico que prefiere evitar complicaciones. Esto hace que a ese profesor se le presente este intento como un una preparación de un modelo innovador. Resulta que es muy fácil proponer juegos a los estudiantes al final de una clase o en algún momento puntual para dinamizar el curso y que puede tener cierta componente motivadora, pero no deja de ser un simple añadido que el estudiante rápidamente entiende como añadido no curricular. En ese caso, las Matemáticas Recreativas, porque quedarían condenadas a ser un mero objeto de distracción del alumnado.

Nuestro punto de vista es que el profesor adquiera la competencia para integrar contenidos de MR con los contenidos de la asignatura matemática. Ahora bien, ¿cómo podemos formar a los docentes para que apliquen la MR desde esta perspectiva? La respuesta puede parecer bastante ingenua, pero adquiriendo las competencias relativas al Diseño de unidades didácticas conteniendo “juegos matemáticos” relacionados con el tema abordado. El medio será elevando a término propuestas de innovación docente al aula, e intentando implicar a otros profesores del centro como proyecto de innovación de centro. Incluso se puede pensar en utilizar o diseñar el uso del ordenador con programas educativo relacionado con la Matemática Recreativa.

Esa es la finalidad del curso que aquí presentamos y que, enmarcado en el ámbito de la innovación docente, pretende formar a los profesores de matemáticas para que aprendan a utilizar las matemáticas recreativas en su labor de un modo creativo e innovador. Por tanto, debe entenderse que el curso no pretende mostrar al docente un conjunto de juegos y acertijos inconexos para ser utilizados en el aula con el fin de motivar o distraer al alumnado, al contrario. El curso es que el profesor aprenda cómo emplear la MR y la adopte una auténtica herramienta didáctica para fomentar la creatividad, mejorar el razonamiento lógico-matemático, despertar la curiosidad y enseñar a pensar a los más jóvenes. Es claro que no centramos en el aprendizaje del cursillista en lugar de intentar enseñar nuestros conocimientos del tema.

\section{Breve descripción del curso}

Este curso lleva más de 15 años impartiéndose, si bien la versión actual del curso está en vigor desde hace cinco años. En esta última etapa el equipo docente está formado por los autores del trabajo.

El curso está destinado principalmente, profesores de Enseñanza Secundaria y profesores Universitarios, si bien pueden inscribirse los profesores de Enseñanza Primaria, estos no son los destinatarios idóneos. Además, este curso está abierto a cualquier estudiante de cuarto curso de grado de estudios científico-técnico y de 
estudios de facultades de Educación y Formación de Profesores, bien sean nacionales o internacionales.

Los objetivos ya se han descrito, aunque los reiteramos nuevamente: Que los docentes interpreten a la MR como un marco de trabajo matemáticas para poder hacer propuestas didácticas personales. Además, que la entiendan como es un lúdico con un gran potencial de impacto didáctico para los estudiantes. Destacamos algunas de características potencialmente didácticas para:

- Inducir al hacer matemático o introducir nuevas ideas.

- Desarrollar diferentes formas de pensamiento.

- Explorar áreas matemáticas menos usuales en la curricula.

- Fomentar la creatividad.

- Modelar matemáticamente el entorno real.

- Analizar y potenciar la precisión del lenguaje.

- Percibir el valor estético del pensamiento matemático.

- Desarrollar estrategias de cálculo mental y computacional.

Para hacer frente a la adquisición de competencias relativas a las características descritas se enumera el siguiente conjunto de contenidos:

1. Juegos: elementos de teoría de juegos.

2. Juegos relativos al cálculo de probabilidades.

3. Acertijos y problemas de lógica e ingenio.

4. Problemas históricos sin solución.

5. Conjeturas matemáticas.

6. Puzles geométricos y aritméticos.

7. Cálculo mental y estrategias de cómputo.

8. Regularidades: sucesiones y series numéricas o gráficas.

9. Problemas de carácter verbal o semántico.

10. Estrategias generales de resolución de problemas.

11. Criptogramas: elementos de teoría de números.

12. Modelización de problemas con un ordenador.

13. Recorridos: cuestiones topológicas y de grafos.

14. La geometría ornamental y modular: teselaciones.

15. Identificación de relaciones geométricas métricas.

16. Cuestiones de género finito: Problemas de género $n$.

17. Problemas abiertos o no resueltos.

18. Utilización de foros de problemas en Internet.

\section{Breve descripción de los temas de contenidos}

En este apartado simplemente hacemos una pequeña sinopsis de los contenidos.

\subsection{Juegos: Elementos de Teoría de Juegos}

Esta rama de las Matemáticas se dedica al estudio de los juegos en que intervienen dos o más jugadores y que se caracterizan además por tener algún tipo de estrategia. 
Mostramos en qué consisten, cuáles son sus metodologías y presentamos algunos de los juegos más tal que entre sus objetos de estudio aparezcan enigmas y juegos que podemos introducir en el aula como MR, por ejemplo, los juegos: "El dilema del prisionero", "El juego de la gallina” y "El dilema de los halcones y las palomas".

\subsection{Juegos relativos al Cálculo de Probabilidades}

Esta área de las Matemáticas que esté directamente relacionada con los juegos, pues el Cálculo de Probabilidades surgió a raíz del estudio de los juegos de azar. En este tema abordamos la MR desde el punto de vista del cálculo de probabilidades, dado que hay bastantes enigmas y acertijos que se nutren de problemas probabilísticos. Por ejemplo, entre los acertijos probabilísticos podemos destacar el "Enigma de las 3 puertas", un ejemplo clásico que es muy didáctico para ilustrar cómo la visión "subjetiva”, o personal, de que cada cual tiene de la probabilidad puede influir negativamente en sus decisiones.

\subsection{Acertijos y problemas de lógica e ingenio}

Los problemas de lógica e ingenio son los problemas tipo que más aparecen en los libros de MR, puesto que son problemas con un enunciado muy atractivo, poseen un gran potencial motivador para aquel que los lee, y necesariamente requieren ralizar una secuencia de razonamientos. En este tema mostramos los clásicos juegos de lógica: los enigmas del tipo “caballeros y escuderos”, los del tipo “muerte o condena” y, por supuesto, las paradojas lógicas. Por ejemplo, de los problemas clásicos de lógica mencionamos el "León y el Unicornio", un enigma aparecido en la obra "Alicia en el país de las maravillas” de Lewis Carroll.

\subsection{Problemas históricos sin solución}

En este tema pretendemos mostrar como los grandes matemáticos de todas las épocas se han esforzado en resolver problemas extremadamente complicados. Por tanto, no se trata de proponer problemas para que los cursillistas los resuelvan. Deseamos que como profesores hagan entender a los estudiante de su labor docente que no todos los problemas tienen solución, y que algunos tiene una enorme dificultad. Sí pues, el tema está dedicado a los problemas matemáticos "sin solución”, como los 23 propuestos por David Hilbert (de los cuáles algunos ya han sido resueltos y otros se encuentran todavía en fase de resolución), los 7 problemas del "Millón de Dólares" de la Fundación Clay (hasta la fecha, sólo ha sido resuelto uno: la "Conjetura de Göldbach") y de aquellos que han pasado a la historia porque han tardado siglos en resolverse, pese a ser abordados por las mentes más brillantes (como el Último Teorema de Fermat).

\subsection{Conjeturas matemáticas}

Este tema está directamente relacionado con el anterior, dado que nos centramos en las conjeturas matemáticas. Por tanto, abordaremos estos dos temas de modo similar, 
centrándonos en este caso en las conjeturas famosas y en las que dejaron de ser conjeturas para convertirse en teoremas tras ser demostradas.

\subsection{Puzles aritméticos y geométricos}

Contenidos clásicos de la MR son los acertijos aritméticos y geométricos. Por ello presentamos en este tema los principales tipos de enigmas geométricos: puzles, particiones, dados, cálculos geométricos.... También, se presentan algunas curiosidades geométricas como las figuras imposibles.

Los problemas aritméticos, presentamos los clásicos enigmas relacionados con repartos, edades, velocidades.... Que, además, pueden ser resueltos empleando técnicas algebraicas. También ¿cómo no? paradojas aritméticas como el problema del "euro perdido".

Tiene cabida en este tema la denominada matemagia, a la cual dedicaremos un apartado, por ser una de las partes de la MR con más llamativa y con más potencial didáctico.

\subsection{Cálculo mental y estrategias de cómputo}

El cálculo mental pasa de estar de moda en el aula en una época a dejar de en la siguiente. En la actualidad no está de moda, sin embargo consideramos que trabajar el cálculo mental y las estrategias de cómputo debe ser una parte favorecida por la labor docente del profesor para afinar el aprendizaje de sus estudiantes. Nada mejor para hacer esto que emplear la MR como medio para enseñar cálculo mental a estudiantes, generando situaciones divertidas donde el estudiante disfrute y aprenda a la vez. En este tema mostramos al docente diversos juegos que pueden emplearse como herramienta didáctica para enseñar a calcular mentalmente.

\subsection{Regularidades: Sucesiones y series numéricas o gráficas}

Este tema es particularmente interesante para los estudiantes de Bachillerato, puesto que en el primer curso se introducen y estudian las sucesiones. Proponemos utilizar para tal fin los problemas recreativos de "La Torre de Brahma" o de la "Leyenda del Ajedrez". Se puede introducir el tema de forma amena y de una manera motivadora. Completar las actuaciones con otros ejemplos de acertijos y enigmas relacionados con series numéricas o gráficas, muy entretenidas, para que los docentes inscritos puedan retar a sus estudiantes en un futuro.

\subsection{Problemas de carácter verbal o semántico}

El lenguaje utilizado por el profesor no es el mismo que el utilizado por el estudiante, y eso genera una dificultad básica de naturaleza lingüística más que matemática. Con este tipo de problemas se fomenta la atención a la capacidad de comprensión de los enunciados que a las características matemáticas en sí. Este tipo de actividades son muy útiles como entrenamiento para aprender a leer y comprender bien los problemas matemáticos, haciendo hincapié en los detalles de los significados de las palabras y del contexto donde están. Se intenta que los estudiantes sean meticuloso a la hora de leer los enunciados de cualquier problema de la materia, que lo entiendan y piensen posibles 
estrategias de resolución, en lugar de intentar resolver los problemas sin tener claro qué se debe hacer.

Con este tipo de juegos se tiene una herramienta para resaltar que el estudiante suele no entienden bien lo que leen, ni siquiera los matices principales de lo que leen. Utilizar este tipo de actividades puede conciencian al estudiante de la necesidad de comprender bien todo lo que leen. Entre los juegos que mostramos como ejemplo tenemos el enigma de "Villavieja y las ovejas", el del "accidente de avión” y otros acertijos del mismo estilo.

\subsection{Estrategias generales de resolución de problemas}

Es muy frecuente que los estudiantes sepan aplicar métodos memorizados para resolver problemas dentro del contexto de la lección que se esté tratando. En muchas ocasiones calculan "mecánicamente” o repiten algoritmos de cálculo de una forma fiable, por ejemplo, saben hacer ecuaciones, derivadas o integrales. ¿Qué sucede cuando deben enfrentarse a un problema que no está en el marco de la lección? ¿Son capaces de aplicar los conocimientos adquiridos para resolver un auténtico problema matemático? Esto es otra cosa. A la posible deficiencia organizadora de los pensamientos de una forma secuencial, suele unirse la dificultad lectora y de comprensión los enunciados, pero todo esto eleva su nivel de dificultad si han de relacionar diferentes áreas de las matemáticas para resolver un problema. Esto se puede resumir con una deficiente actitud para plantear los problemas, y aquí es donde el profesor de matemáticas una gran resistencia pasiva ante el método de enseñar a resolver problemas. Por ello se dedica este este tema a las estrategias de resolución de problemas con una perspectiva distinta. Mostramos a los docentes cómo pueden entrenar a MR. Al fin de cuentas, los problemas matemáticos "serios" y los enigmas recreativos tienen muchos rasgos comunes: la dificultad, el reto de no ser algo mecánico; sin embargo, los acertijos son más atractivos para los estudiantes que los problemas matemáticos serios.

\subsection{Criptogramas: Elementos de Teoría de Números}

Podríamos definir, de una forma clásica, la criptografía como el arte de escribir un texto en clave. La práctica criptográfica fue empleada en la antigüedad en Egipto y en Mesopotamia haciendo uso de unos alfabetos muy gráficos. Hoy podemos entender los de entonces como unos métodos de cifrado que hoy traducimos a símbolos alfabéticos. Son los romanos quienes emplearon el arte de codificar las palabras construidas con caracteres alfabéticos actuales para comunicarse en el ámbito militar sin que se pudiera romper el secreto de lo escrito, para ello basta hacer referencia al Cifrado Cesar.

Actualmente la criptografía es una técnica directamente relacionada con diversos campos de las matemáticas como la estadística, la aritmética modular y la teoría de números. Además, en la sociedad de la información y la comunicación, en que internet se ha adueñado de todo, en que nuestros datos personales, profesionales, médicos, bancarios están almacenados en nuestras computadoras personales o en las de nuestro banco, la seguridad informática se ha vuelto una auténtica prioridad.

La criptografía es especialmente importante en las ciencias de la computación, para transmitir la información de forma segura. Por ello, en este tema presenta en qué consiste la criptografía, en general y los criptogramas, en particular, así como y los 
principios básicos de cifrado. Se muestran enigmas y acertijos de MR relacionados con la temática propuesta, para introducir a los inscritos en el mundo de la codificación de forma amena y motivadora.

\subsection{Modelización de problemas por ordenador}

La modelización matemática consiste básicamente en traducir un problema del mundo real, al lenguaje matemático. Una vez resuelto a nivel matemático y evaluados los resultados obtenidos, se ha de volver a traducirlos en términos de problema inicial real. En algunos casos, este proceso de modelización matemática suele ser asistido con ordenador que resultar un gran herramienta. Al tener mecanizado sobre el ordenador el modelo matemático, entonces se pueden realizar múltiples simulaciones informáticas del problema que se intenta resolver. En el ámbito de la educación, el aplicación de geometría dinámica, Geogebra, posibilita al docente diseñar modelizaciones dinámicas para resolver problemas matemáticos.

Un clásico problema matemático-recreativo que fue demostrado en 1976 únicamente gracias a la potencia del ordenador fue el famoso "Problema de los cuatro colores": ¿Cuántos colores son suficientes para colorear un mapa adecuadamente? En otras palabras, de modo que dos países con frontera común tengan colores distintos.

\subsection{Recorridos, cuestiones topológicas y de grafos}

La teoría de grafos es una rama de las matemáticas que surgió a raíz de lo que podríamos considerar un problema recreativo del mundo real, el famoso problema de los “Puentes de Könisberg”, resuelto por el célebre matemático Leonard Euler en 1736:

El rio Pregel a su paso por la ciudad de Könisberg se bifurcaba en varios ramales formando dos islas, que estaban conectadas entre sí y con las orillas del rio por 7 puentes. A lo largo de los años los habitantes de Könisberg trataron de encontrar una ruta que permitiera pasar por todos los puentes sin pasar dos veces por el mismo y no lo lograron.

Euler demostró que era imposible realizar tal recorrido, sentando las bases de una nueva rama de las matemáticas, la Topología y dentro de esta, la Teoría de Grafos.

Este es el caso más claro de cómo la matemática seria puede surgir a partir del estudio de problemas recreativos.

El matemático y divulgador, Miguel de Guzmán comentaba al respecto:

"Hay igualmente una componente lúdica posible, que podrá extrañar a algunos, pero es un hecho profundo que una gran parte de la matemática más seria ha sido desarrollada bajo una motivación inicial lúdica y que los puzles y juegos mentales comparten con la matemática muchos procesos de pensamiento eficaces en uno u otro campo. Es más, muchas veces resulta extraordinariamente difícil decidir dónde termina el juego y dónde comienza la actividad científica 'seria'. Para muchos de los matemáticos, la matemática nunca deja del todo de ser un gran juego.” 
En este tema tras hablar del famoso problema, presentamos brevemente en qué consisten la topología y la teoría de grafos y cómo surgieron a partir de este simple “enigma” y, por supuesto, veremos qué tipos de acertijos y enigmas están relacionados con este tema (por ejemplo, el clásico "dibujar un sobre sin levantar el lápiz del papel”)

\subsection{La geometría ornamental y modular: teselaciones}

Una teselación del plano consiste en rellenarlo por completo, usando como piezas figuras planas cumpliendo las siguientes condiciones: No pueden quedar espacios entres las figuras (es decir, el plano debe quedar cubierto por completo), y no se pueden superponer las piezas utilizadas.

En este tema se abordan diversos problemas de MR de teselaciones y se ofrecen indicaciones para relacionarlos con la geometría en el aula. Entre las actividades que no pueden faltar en un tema sobre la geometría ornamental y modular, está analizar o diseñar actividades relacionadas con la obra de M.C. Escher, que conjuga arte y geometría, belleza y matemática; un deleite matemático para los sentidos y para despertar la intuición geométrica en el aula.

\subsection{Identificación de relaciones geométricas métricas}

Las relaciones geométricas métricas son otra gran fuente de problemas matemáticos y de acertijos y enigmas de matemáticas recreativas. Además, se puede transformar cualquier problema geométrico en un problema recreativo, para ello basta escribir un enunciar de modo diferente. También se puede emplear programas de geometría dinámica (Geogebra) para transformar el problema en un aplicación interactivo. Esto último permite motivador a los estudiantes. Así pues, en este tema hablamos de las relaciones geométricas métricas y mostramos ejemplos de problemas recreativos relacionados con la geometría métrica.

\subsection{Cuestiones de género finito: Problemas de género n}

Este tema está dedicado a la aplicación de la Matemática Discreta en el ámbito de las MR. La Matemática Discreta es la rama de las matemáticas dedicada al estudio de los conjuntos finitos o de los conjuntos numerables. La matemática discreta aborda temáticas como la teoría de conjuntos, la lógica matemática y la combinatoria que aparecen en un sinfín de juegos. Por tanto, presentamos algunos juegos relacionados con cada una de ellas y propondremos diferentes actividades para ampliar el tema.

\subsection{Problemas abiertos o no resueltos}

Se denominaremos problema abierto a un problema que no está completamente resuelto. En muchos casos es porque de algún modo faltan datos o detalles para poder resolverlo. Nuestros estudiantes se enfrentan a ellos, investigando, buscando la información necesaria, formulando sus propias teorías, conjeturas, hipótesis...

En MR podemos proponer problemas abiertos para incentivar que los estudiantes desarrollen su creatividad, sus capacidades de investigación, su razonamiento... 
Entre los problemas abiertos más entretenidos se presentan aquellos que son denominados como problemas de pensamiento lateral. Se la muestra al estudiante una situación problemática una historia y tienen que descubrir qué ha sucedido en esa historia. El estudiante sólo puede formular preguntas concretas, en que el profesor y éste sólo contesta: o sí o no o indiferente. Este tipo de problemas son interesantes porque inician al estudiante a investigar en una situación completamente incomprensible en un principio buscando las preguntas que debe formularse.

\subsection{Utilización de colecciones de problemas en Internet}

En este tema relacionamos algunas direcciones y foros, así como, la forma de emplearlos para poder usar elementos de la MR en la actuación docente dentro del aula. En Internet se pueden encontrar diferentes páginas web, blogs, etc...dedicados a los juegos matemáticos, en que se plantean enigmas que se debaten y se resuelven a través de foros de discusión Por ejemplo, la lista de Snark: dedicada al intercambio y resolución de recreaciones matemáticas, o el blog Gaussianos dedicado a las matemáticas, no sólo a la MR.

\section{Metodología}

Para enfocar el curso en la línea innovadora que pretendíamos, decidimos diseñar nuevos materiales didácticos y utilizar las nuevas tecnologías como herramienta para dinamizar el curso y lograr una participación activa en el mismo por parte de los estudiantes (profesores o futuros profesores).

\section{Materiales didácticos}

Guía del estudiante: Esta amplia guía consta de dos partes diferenciadas. En la primera parte con toda la información del curso y la organización del mismo (metodología, temporalización, evaluación, equipo docente...). En la segunda se ofrece una visión general del curso, tema por tema, describiendo los contenidos y tipo de problemas que tratan en cada uno de ellos.

Guías Didácticas: Para cada uno de los 18 temas del curso está diseñada una guía didáctica dónde, además de varios problemas recreativos, se encuentran reseñas históricas, relación del tema con el mundo real, curiosidades para comentar en el aula y una sección en que presentamos películas, artículos o documentales relacionados con la temática, para acercar a los estudiantes al mundo real.

Retos Quincenales: Una vez comenzado el curso, con una periodicidad de dos semanas se plantea un reto en un foro del curso virtual. Tales retos suelen constar de diversos problemas de matemática recreativa, cuidadosamente seleccionados y presentados de forma atractiva. Solemos empezar con uno sencillo, que se va complicando gradualmente. También, se proponen retos de problemas normales que pueden ser interpretados con un enunciado adecuado como problema recreativo. 
Por otro lado, el enunciado del reto quincenal suele incluir una parte de didáctica de las matemáticas, orientando y proponiendo a los estudiantes del curso que diseñen actividades con el objetivo de introducir esos acertijos en el aula donde son profesores.

El reto quincenal es voluntario, no es necesario que participen para superar el curso, pero se comenta que es muy recomendable la participación en los mismos por el poder formativo que tienen al ser prácticas claras. Por un lado, logramos dinamizar el curso y los foros se transforman en una zona de debate y trabajo colaborativo en que los estudiantes aportan sus ideas, colaboran unos con otros, se divierten jugando y por el otro, evaluamos el potencial interés de los alumnos por el curso.

Las soluciones a los diferentes retos sólo se envían a aquellos que han participado en los mismos por correo electrónico. Los retos quedan abiertos desde que se cuelgan y los estudiantes pueden enfrentarse a los mismos en cualquier momento del curso, lo que permite que adapten su dedicación en función de su disponibilidad horaria.

\section{Curso virtual}

La Metodología que hemos aplicado en este curso es la propia de la enseñanza a distancia actual que brinda toda una serie de posibilidades didácticas impensables hace tan sólo unos años. Concretamente en las dos últimas ediciones del curso, hemos utilizado la plataforma virtual de cursos de la UNED.

En el entorno telemático (virtual) se distinguen las diferentes herramientas:

- Entorno de trabajo: Zona virtual de la asignatura en la plataforma actual (Alf) de la UNED.

- Herramientas de comunicación personal: La UNED dota de cuenta de correo a cada una de las personas que forman la comunidad académica. El correo electrónico se suele utilizar para comunicaciones personales que no deben ser vistas por otros participantes.

- Herramientas de comunicación colectiva: La estructura de foros personales permite hacer un seguimiento de todos los mensajes del estudiante y las respuestas del equipo docente. El foro de comunicaciones del equipo docente permite clarificar cuestiones y aportar la documentación complementaria insertando esta información como ficheros adjuntos a los mensajes.

Esta estructura de foros personalizados ya lo aplicamos anteriormente y de forma exitosamente en una asignatura del Máster de Formación del Profesorado (Innovación Docente e Iniciación a la Investigación Educativa). Decidimos aplicar el mismo modelo de comunicación para el curso de matemática recreativa. Esta modalidad permite la comunicación bidireccional estudianteprofesor a modo de diario de bitácora, además, todos los estudiantes pueden ver los foros de sus compañeros, pero no pueden insertar mensajes en foros ajenos. Todos tienen acceso de lectura escritura en el foro de consultas generales.

- Herramientas de webconferencias: Con esta herramienta pueden generarse videoconferencias que los estudiantes pueden ver en directo o en diferido. 


\section{Duración del curso}

El curso tiene una duración semestral (del 1 de diciembre al 31 de mayo) y tiene prevista una dedicación de alrededor de 150 horas.

\section{Evaluación}

Para superar el curso el alumno deberá confeccionar y enviar sólo una de las siguientes propuestas:

1. Uno o dos trabajos en donde se analizará y desarrollará un método de impartir un tema de los propuestos. Se valorará es la viabilidad de ejecutarlo en el aula de Matemáticas.

2. Un trabajo de innovación educativa donde exista una propuesta relacionada con la Matemática Recreativa.

3. Un trabajo donde el vehículo curricular sea algún aspecto de la Matemática Recreativa. desarrollo de software con talante educativo

\section{Resultados}

Una vez superado el curso los profesores (o futuros profesores) comprenden que la MR se puede emplear en el aula con relativa facilidad superando su carácter lúdico y potenciando su carácter motivador. Además, cuentan con las herramientas y materiales necesarios para integrar de forma coherente la Matemática Recreativa en sus prácticas educativas e incluso para desarrollar, e iniciar, algún proyecto de Innovación Docente relacionados con la MR.

Pero no sólo aprenden a emplear la MR como herramienta didáctica, sino que ellos mismos se ven envueltos en un entorno de aprendizaje en el que la MR es el pilar fundamental y en el que jugar y resolver retos para ir avanzando en el curso es un requisito.

Aquellos profesores que aprovechan el curso para poner en práctica lo aprendido en el aula, comentan cómo han disfrutado sus estudiantes, como ha mejorado el ambiente en el aula, cómo han motivado a sus jóvenes alumnos y cómo la matemática recreativa les ha permitido introducir prácticas educativas innovadoras en el aula.

\section{Conclusiones}

Si comparamos nuestro curso desde que decidimos realizar las innovaciones aquí presentadas con las antiguas ediciones del mismo, que se limitaban a enviar material didáctico a los estudiantes, que no utilizaban el curso virtual y que, por tanto, no constaban con la interacción constante entre estudiantes y equipo docente la conclusión es clara: estamos en el camino correcto.

Pero no por estar haciendo un buen trabajo, dejaremos de mejorar. Nosotros creemos en la mejora continua, por tanto, en cada nueva edición del curso valoramos los puntos positivos y negativos de la edición anterior con vistas a mejorarlo. 
Para próximas ediciones tenemos en mente realizar mini-videos para plantear los retos y las soluciones a los mismos de un modo más cercano, buscar fórmulas para incrementar la participación activa de los estudiantes en los foros, involucrar a los más interesados en proyectos de innovación docente relacionados con la matemática recreativa, utilizar las redes sociales para difundir el curso y para crear una red de profesores y estudiantes interesados en la matemática recreativa, diseñar una página web o un blog para colgar los materiales generados por nuestros alumnos y desarrollar un curso MOOC, como escaparate donde mostrar lo que hacemos en el curso oficial.

\section{REFERENCIAS}

- Echenique, I. (2006): “La resolución de problemas matemáticos”. Fondo de publicaciones del gobierno de Navarra.

-de Guzmán, M. (1994). “Para pensar mejor. Desarrollo de la creatividad a través de los procesos matemáticos”. Madrid. Ediciones Pirámide.

-de Guzmán, M. (1984). “Cuentos con cuenta” Editorial Labor Bolsillo Juvenil.

-de Guzmán, Miguel (1984): “Juegos matemáticos en la enseñanza”

Publicado en las IV Jornadas sobre Aprendizaje y Enseñanza de las Matemáticas.

-de Guzmán, Miguel (1983): “Aspectos insólitos de la actividad matemática”

Artículo publicado en la revista Investigación y Ciencia. Febrero 1983, pp. 100-108

- Polya, G. (1989): “Cómo plantear y resolver problemas”. México. Editorial Trillas.

-Deulofeu: "Prisioneros con dilemas y estrategias dominantes. Teoria de Juegos"

Colección "El mundo es matemático”. RBA (2010).

-Corbalán, Fernando: "La proporción aúrea. El lenguaje matemático de la belleza”

Colección "El mundo es matemático”. RBA (2010).

-Loyd, Sam: "Los acertijos de Sam Loyd”

Biblioteca Desafíos Matemáticos. RBA (2007).

-Loyd Sam: "Nuevos acertijos de Sam Loyd”

Biblioteca Desafíos Matemáticos. RBA (2007).

-Dudeney, Henry E: “El acertijo del Mandarín (Diversiones Matemáticas I)”.

Biblioteca Desafíos Matemáticos. RBA (2007).

-Emmet, Eric: "Juegos para devanarse los sesos”

Biblioteca Desafíos Matemáticos. RBA (2007)

-Gardner, Martín: “¡Ajá! Paradojas que hacen pensar” 
Biblioteca Desafíos Matemáticos. RBA (2007).

-Gardner, Martin: “¡Ajá! Inspiración!”

Biblioteca Desafíos Matemáticos. RBA (2007).

-Gardner, Martin: "Viajes por el tiempo y otras perplejidades matemáticas” Biblioteca Desafíos Matemáticos. RBA (2007).

-Paenza, Adrián: “Matemática, ¿estás ahí?”

Biblioteca Desafíos Matemáticos. RBA (2007).

-Recamán, Bernardo: "Las nueve cifras, el cambiante cero y otros divertimentos matemáticos”. Biblioteca Desafíos Matemáticos. RBA (2007).

-Smullyan, Raymond: “¿Cómo se llama este libro?”

Biblioteca Desafíos Matemáticos. RBA (2007).

-Stewart, Ian: "Ingeniosos encuentros entre juegos y matemática” Biblioteca Desafíos Matemáticos. RBA (2007). 